\title{
Overhead Reduction and Performance Enhancement of AODV and DSR Routing Protocols
}

\author{
Prabhat Kumar Khemariya \\ Department of Electronics \& Communication \\ Engineering \\ Sagar Institute of Research, Technology and \\ Science Bhopal, India
}

\author{
Jyoti Jain \\ Department of Electronics \& Communication \\ Engineering \\ Sagar Institute of Research, Technology and \\ Science Bhopal, India
}

\begin{abstract}
Mobile Ad hoc Network is an infrastructure less multi hop wireless network. In this network a node communicates directly with other mobile nodes within the network range. Transmission power of the nodes plays an important role here. The objective of this work is to study the behavior and performance of the three frequently used MANET routing protocols with respect to different transmission power of individual nodes of the network, these are Dynamic source routing (DSR), ad hoc on demand distance vector (AODV with HELLO message) and AODV (without HELLO message). Analysis shows the positive and negative impact of increase in transmission power of individual nodes on the performance of these routing protocols. The outcomes of the study are more significant and can be used in the design of new power aware routing protocols.
\end{abstract}

\section{General Terms}

Transmission power, network range

\section{Keywords}

Overhead, transmission range, MANETs, Routing protocols

\section{INTRODUCTION}

Wireless communication is an emerging and upcoming technology that will allow users to access information and services electronically, irrespective of their geographic location. There are solutions to these demands, one being wireless local area network. However, there is increasing demand for connectivity in places where there is no infrastructure available. This is where ad hoc network came into existence (1).

Mobile ad-hoc network are autonomously self-organized and self configuring networks without infrastructure support. In such networks, since node mobility is very high the network may experiences frequent and unpredictable topology changes. Mobility and the absence of any fixed infrastructure make mobile ad-hoc networks very attractive for time critical applications.

The infrastructure less and the dynamic nature of these networks demand new set of networking strategies to be implemented in order to provide efficient end to end communication. MANETs can be deployed quickly at a very low cost and can be easily managed. In the future, there is no doubt there will be more ad hoc networks, in which overhead in routing protocols is one of the critical issue.

The node mobility that causes frequent link failures which result in serious performance degradation and causes overhead. The links in MANET are mostly unidirectional and the node transmission power is affected by interferences. So the nodes use different transmission powers. In multi hop networks in variable power scenarios, where the nodes can dynamically vary their transmitter power levels. (2) In such cases, greater energy efficiency can be obtained if the nodes choose the transmission power depending on the distance between the transmitter and receiver nodes (3). The transmission range of the node is a very important aspect which will have more influence on the network connectivity. The nodes with sufficiently higher transmission range can maintain connectivity even at higher mobility. In a multi hop communication scenario, there may be frequent link failures because of rapid change in topology due to node mobility. If we increase the transmission range of power to increase the one hop distance of the nodes, then it will certainly avoid link failures due to mobility to some extent. Here, the work has been done for reducing overhead by varying transmission power or transmission range for DSR and AODV Routing protocols.

\section{ROUTING PROTOCOLS}

The inadequate and limited resources in MANETs have made designing of an efficient and reliable routing strategy a very challenging task. An intelligent routing algorithm is required to efficiently use these limited resources while at the same time being adaptable to the changing network conditions such as network size, traffic density, nodes mobility, network topology and broken routes.

Numerous routing protocols have been proposed and developed for ad hoc networks. Such protocols must deal with the limited resources available with these networks, which include high power consumption, low bandwidth and high mobility. Existing routing protocols can be classified in many ways, but most of these are done depending on routing strategy and network structure. (4)

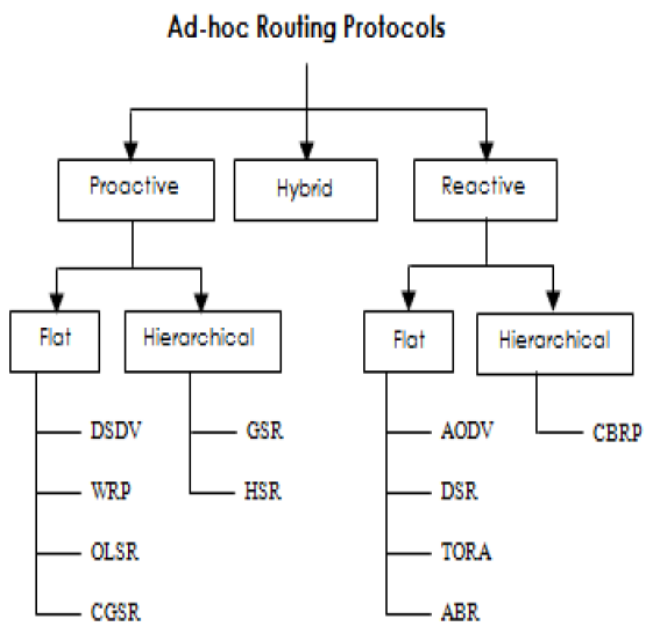

Fig. 2.1: classification of routing protocols (5) 
DSR and AODV routing protocols are reactive type and they provide more efficient routing in MANET.

\subsection{DSR routing protocol}

DSR gives rise to a completely self organizing and self configuring network without the need for any existing network infrastructure or administration. It uses Route Discovery and Route Maintenance mechanisms to discover and maintain source routes to arbitrary destinations. (6)

When a source node wants to send a packet, it first searches for an entry in its route cache. If the route is available, the source node includes the routing information inside the data packet before sending it. Otherwise, the source node initiates a route discovery operation by broadcasting route request (RREQ) packets. Each RREQ packet is uniquely identified by the source address and the request id (a unique number). On receipt it the RREQ packet, an intermediary node checks its route cache. If the node doesn't have routing information for the requested destination, it appends its own address to the route record field of the route request packet. Then, the request packet is forwarded to its neighbors. A node processes route request packets only if it has not seen the packet before and its address is not presented in the route record field. If the route request packet reaches the destination or an intermediate node has routing information to the destination, a route reply packet is generated. When the route reply packet is generated by the destination, it comprises addresses of nodes that have been traversed by the route request packet. Otherwise, the route reply packet comprises the addresses of nodes the route request packet has traversed concatenated with the route in the intermediate nodes. (4)
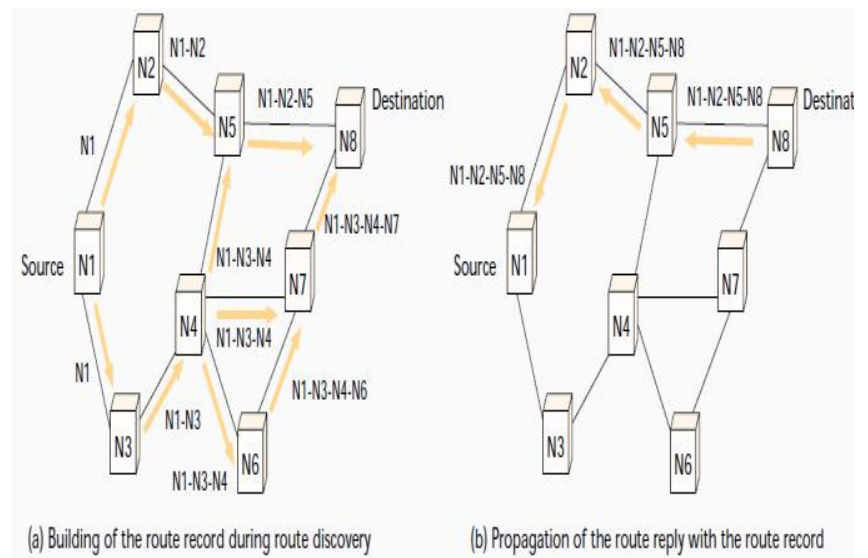

(a) Building of the route record during route discovery

Figure 2.2: DSR routing protocol (5)

\subsection{Ad-Hoc on Demand Distance Vector (AODV) Routing protocol}

AODV is a reactive routing protocol and the routes are calculated only when needed. It maintains these routes as long as they are needed by the source. It uses trees to connect multicast group members. It is self-starting, loop free and highly scalable. It supports both unicast and multicast routing (7) $(8)$.When a source node wants to send packets to the destination, it initiates a route discovery operation if no route is available. In the route discovery operation, the source broadcasts route request (RREQ) packets. A RREQ includes addresses of the source and the destination, the broadcast ID, which is used as its identifier, the last seen sequence number of the destination as well as the source node's sequence number. Sequence numbers ensure loop-free and up-to-date routes. (4)

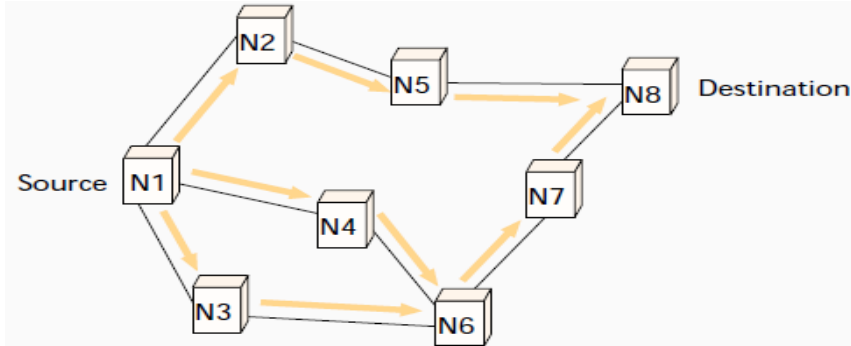

(a) Propagation of the RREQ

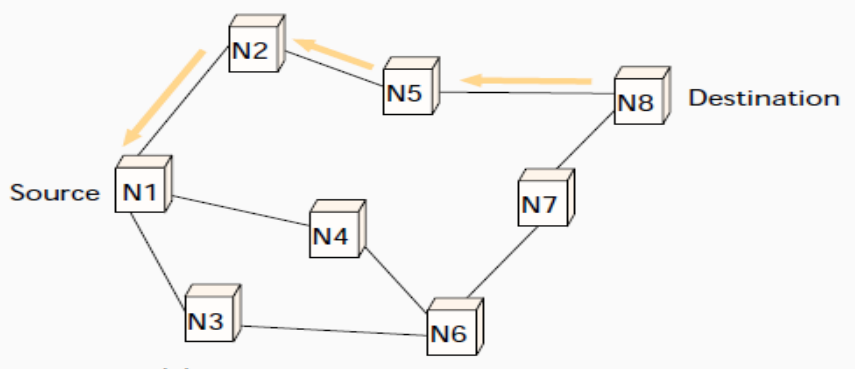

(b) Path of the RREP to the source

Figure 2.3: AODV routing protocol (5)

\section{LITERATURE SURVEY}

I.Srinivas sethi, Ashima rout An Effective and Scalable AODV for Wireless Ad hoc Sensor Networks. In this paper, to reduce time delay for delivery of the data packets, routing overhead and improve the data packet delivery ratio. For this, a third party reply model algorithm was developed and simulated by NS-2 under Linux operating system. The performance of routing protocol was evaluated under various mobility rates and found that the proposed routing protocol is better than AODV.

II. Xuefei Li and Laurie Cuthbert On-demand Node-Disjoint Multipath Routing in Wireless Ad hoc Networks. An on demand Node Disjoint Multipath Routing protocol is proposed to overcome the draw backs of routing protocols like AODV and DSR. The protocol has novel aspects are, it reduces routing overhead and achieve lower data delay as well as higher packet delivery ratio.

III. Swarnali Hazra and Sanjit Setua Optimization on Control Overhead in MANET. The transmission overhead is reduced by stopping the unnecessary broadcasting of Route Request packets and unnecessary traversing of Route Reply packets. And proposed the optimized DSR and optimized AODV routing protocols by specific algorithm.

IV. Xian Ming Zhang, En Bo Wang, Jing Jing Xia, and Dan Keun Sung An Estimated Distance-Based Routing Protocol for Mobile Ad hoc Networks. In this paper, an estimated distance (EstD) based routing protocol (EDRP) proposed to steer a route discovery in the general direction of a destination, which can restrict the propagation range of route request (RREQ) and reduce the routing overhead and improve the routing performance in dense or high mobility networks.

V. Li Xia, Zhaohui Liu, Yue Chang, Peng Sun An Improved AODV Routing Protocol Based on the Congestion Control and Routing Repair Mechanism. Proposed an improved AODV protocol called AODV-I. By adding the congestion processing and the routing repair mechanism to the RREQ message, the new protocol reduces the packet loss rate, end- 
to-end latency and enhances the utilization rate of the network resources.

VI. Ambarish R. Bhuyar and V. T. Gaikwad Reducing Routing Overhead in Mobile Ad Hoc Network using Probabilistic Rebroadcast Mechanism. Probabilistic rebroadcast mechanism is used to reduce overhead, In which rebroadcast delay is introduced to determine the neighbor coverage knowledge which will help in finding accurate additional coverage ratio and rebroadcast order.

VII. V. Lalitha and R.S. Rajesh The Impact of Transmission Power on the Performance of MANET Routing Protocols. The multi hop routing protocols deliver acceptably good performance only at a particular levels of transmission powers, and the use of high transmission power will reduce lot of overheads and gives better performance.

\section{PROPOSED WORK}

In this paper, the overhead calculation and reduction has been done with varying Transmission power on the constant node speed i.e. $10 \mathrm{~m} / \mathrm{s}$.

In MANET transmission range of the network increases with transmission power, when the topology changes in multi hop network, in that condition if transmission power increases, it will able to transmit the message in wide range and it will certainly avoid the link failure, in this condition overhead of the network will reduce.

The free space model (9)assumes the ideal propagation condition of clear line of sight path between the transmitter and receiver. The equation for this calculation by Friis (10) is given by -

$$
\frac{P r}{P t}=\operatorname{GtGr}\left(\frac{\lambda}{4 \pi r}\right)^{2}
$$

Here,

$\operatorname{Pr}=$ received power

$\mathrm{Pt}=$ transmitted power

$\lambda=$ wave length

$\mathrm{g}=$ gain

$\mathrm{r}=$ transmission range

Here, the transmission power of the network increased for the same node speed and number of nodes, and overhead calculation have been done, to find out the best transmission power in which less overhead occur. There are some factors like RRQ, RREP and RERR which are responsible to increase overhead in the ad hoc network, on different values of transmission power the overhead calculated with these factors.

When one node needs to send a message to another node for which it does not already have a route it broadcasts a Route Request (RREQ) message. By sending RREQ the node is asking the network how to get to the destination. The RREQ message contains several key bits of information like the source, the destination, the lifespan of the message, broadcast ID etc, when a data packet carries all these information in its header file it increases the data size and because of it overhead increases.

In RREQ message arrives at its destination, a "Route Reply" (RREP) message will immediately get passed back to the origin, indicating that a route to the destination was found. but sometimes RREP message does not reaches to the source and in that condition source again send the same data which previously send to the destination so the overhead increases because of it.

When data packet transfer from source to destination by a particular route and the route has broken, then data has been lost and communication does not exist between the source and destination and "Route error" (RERR) generated. Route error is also a main cause of improving overhead conditions.

Here, these conditions are observed and a calculation have been done to face these problems and to reduce the overhead.

When the data are send from the source to the destination, a transmission power is needed which transmits the data, which are calculated at different points Under these conditions, AODV was also calculated with HELLO message and without HELLO message.

\section{SIMULATION PARAMETERS}

In constant node speed, the transmission power are varied for DSR and AODV (with HELLO message and without HELLO message) at physical layer in Qualnet simulator to find out the best transmission power which reduces the overhead. The simulation has been done on the transmission power $12,13,15,16$ and $18 \mathrm{mbps}$, for the constant node speed i.e. $10 \mathrm{~m} / \mathrm{s}$.

Table 1: simulation parameters

\begin{tabular}{|l|l|}
\hline Simulation parameters & \multicolumn{1}{|c|}{ Value } \\
\hline Simulator & Qualnet 5.2 \\
\hline Topology size & $1500 * 1500$ \\
\hline Number of nodes & 75 \\
\hline Transmission power & $12,13,15,16,18 \mathrm{mbps}$ \\
\hline Traffic type & CBR \\
\hline No. of CBR connection & 25 \\
\hline Packet size & $512 \mathrm{byte}$ \\
\hline Pause time & $30 \mathrm{sec}$ \\
\hline Node speed & $10 \mathrm{mps}$ \\
\hline
\end{tabular}

\section{RESULTS \& ANALYSIS}

Here, the calculations have been done with different overhead causing factors, which are listed as number of route request packet initiated, number of route repeat packet initiated, number of route repeat packet initiated as intermediate nodes, number of route repeat initiated as destination. The final calculation is done by adding values of above stated factors for the different routing protocols.

In figure 6.1 , the number of "route request packet initiated" are shown for DSR, AODV (With HELLO message) and AODV (without HELLO message) on the Transmission power $12,13,15,16$ and 18 . 


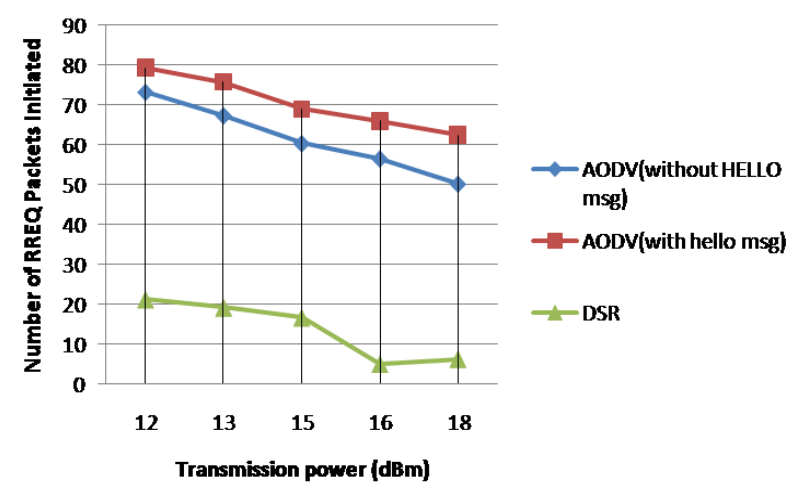

Figure 6.1 number of RREQ packets initiated

The following figure 6.2 shows the calculation for route error packet initiate for routing protocols

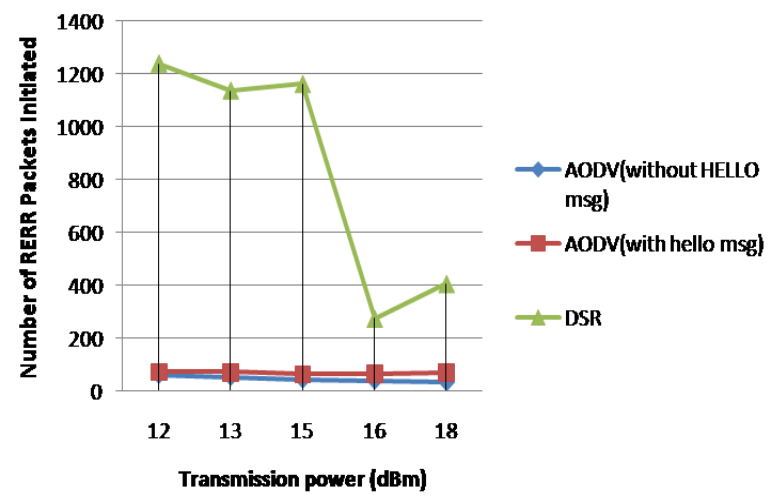

Figure 6.2 number of route error packets initiated

Following figure 6.3 shows the number of route repeat packet initiated as intermediate node

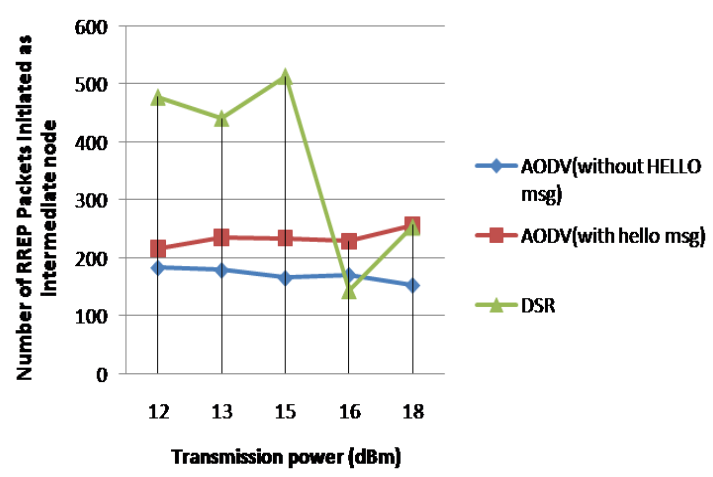

Figure 6.3 Number of RREP packet initiated as intermediate node

In the following figure 6.4, calculation is done for the number of route repeat packets initiated as destination

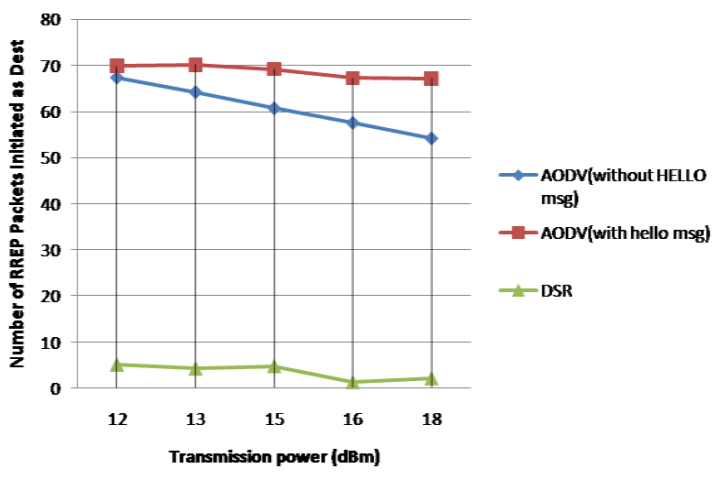

Figure 6.4 Number of RREP packets initiated as destination

Following figure 6.5 shows the final calculation of the overhead and the value of the best transmission power for the routing protocols DSR, AODV (with HELLO message) and AODV (without HELLO message).

Table 2: Calculations of different RP (routing protocol)l on different Transmission power

\begin{tabular}{|l|l|l|l|}
\hline & $\begin{array}{l}\text { AODV } \\
\text { (without } \\
\text { TELLO } \\
\text { message) } \\
\text { power }(\mathrm{dBm})\end{array}$ & $\begin{array}{l}\text { AODV } \\
\text { (with } \\
\text { HELLO } \\
\text { message) } \\
\text { Byte }\end{array}$ & DSR (Byte) \\
\hline 12 & 384.4063 & 437.5997 & 1741.854 \\
\hline 13 & 363.8799 & 453.1734 & 1600.403 \\
\hline 15 & 329.3864 & 437.7069 & 1695.427 \\
\hline 16 & 323.6 & 428.6667 & 425.6933 \\
\hline 18 & 289.2533 & 456.64 & 668.64 \\
\hline
\end{tabular}

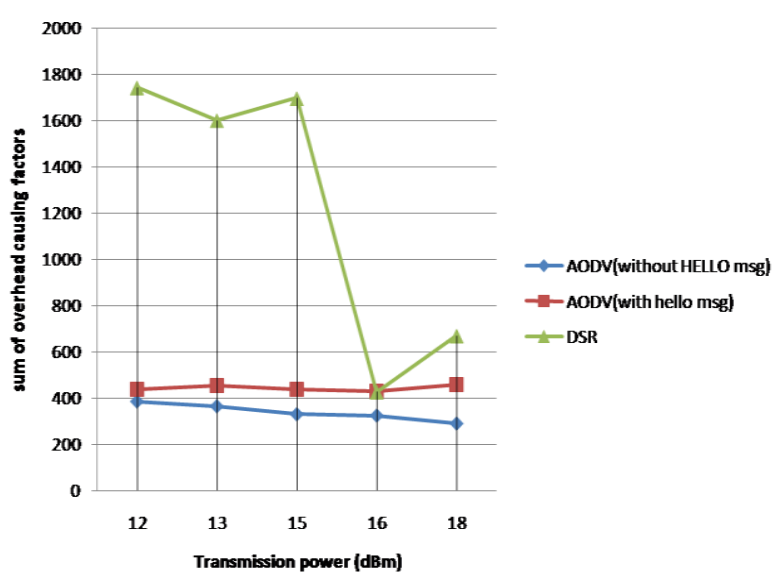

Figure 6.5 final calculation of best transmission power for routing protocols 
Final result is based on the addition of the overhead causing factors i.e., number of route request packet initiated, number of route repeat packet initiated, number of route repeat packet initiated as intermediate nodes, number of route repeat initiated as destination.

Based on the above stated Factors, the simulation results show that $16 \mathrm{dBm}$ is the best transmission power for DSR routing protocol and AODV (with hello message), and $18 \mathrm{dBm}$ is the best transmission power for AODV (without HELLO message). So the resultant value of best transmission power in MANET is $16 \mathrm{dBm}$, because on this value both the protocol (DSR, AODV with hello message) are working with less overhead.

\section{CONCLUSION}

Here, successfully evaluated the performance of routing protocols i.e. DSR, AODV (with HELLO message) and AODV (without HELLO message) under different levels of transmission power of the nodes. Obviously the change in transmission power has a significant impact on the performance of the routing protocols. The multi hop routing protocols deliver acceptably good performance only at a particular levels of transmission powers. Even though the use of "high" transmission power will reduce lot of overheads and give excellent performance.

If we use the minimum transmission power in the network, it will increase the hops and so considerably increase the overhead and reduce the performance of the network.

These results shows that, DSR and AODV (with hello message) protocols works efficiently on or before the specific transmission range which is $16 \mathrm{dBm}$, so we can consider it the best transmission power for these routing protocols. In a general sense, that "minimum" transmission power has a very lower transmission range, but in that situation if we convey the message in wide area then it will cause more overhead and poor performance of the network. So here, the high transmission power used for the better performance of the network.

\section{REFERENCES}

[1] DOA: DSR over AODV Routing for Mobile Ad Hoc Networks. Singha, Rendong Bai and Mukesh. s.l. : IEEE TRANSACTIONS ON MOBILE COMPUTING, 2006, IEEE Transection, Vol. 5.

[2] The Impact of Transmission Power on the Performance of MANET Routing Protocols. V.Lalitha, Dr.R.S.Rajesh. 2, FEB 2013, IOSR Journal of Engineering (IOSRJEN), Vol. 3, p. 34.

[3] Highly dynamic Destination-Sequenced Distance-Vector routing (DSDV) for mobile computers. Bhagwat, Charles E. Perkins and Pravin. August 1994. SIGCOM 94 Conference on Communications Architecture. pp. 234244.

[4] Mobile Ad Hoc Network Routing Protocols:A Comparative Study. Charu Wahil, Sanjay Kumar Sonbhadra2. no. 2, Noida : International Journal of $\mathrm{Ad}$ hoc, Sensor \& Ubiquitous Computing (IJASUC), 2012, Vol. Vol.3.

[5] A Review of Current Routing Protocols for Ad Hoc Mobile Wireless Networks. Royer, Elizabeth M. California : IEEE Personal Communications, 1999.

[6] Sandeep Sahu, Sonam Jain. Geometric Routing Protocol based on Genetic Algorithm for Minimized Delay in MANETs. s.l.: International Journal of Electronics Communication and Computer Technology (IJECCT), May 2012. Vol. 2.

[7] The effects of on-demand behavior in routing protocols for multi-hop wireless ad hoc networks. D.A. Maltz, J. Broch, J. Jetcheva and D.B. Johnson. s.l. : IEEE, August 1999.

[8] D.A.Maltz, D.B. Johnson and. Dynamic source routing in ad hoc wireless networks. s.l. : Mobile Computing, Kluwer Academic Publishers,, 1996. pp. 153-181.

[9] The Dynamic Source Routing Protocol for Mobile Ad hoc networks. Josh Broch, David B. Johnsson, David A. Maltz,. March 1998.

[10] A Note on a Simple Transmission Formula. Friis, H. T. s.l. : Proc. IRE, May 1946, Vol. 34 